\title{
Morphology-Conductivity Relationship in Crystalline and Amorphous Sequence-Defined Peptoid Block Copolymer Electrolytes
}

\author{
Jing Sun, ${ }^{\dagger}$ Xunxun Liao, ${ }^{\ddagger}, \|$ Andrew M. Minor, ${ }^{\ddagger}, \|$ Nitash P. Balsara, ${ }^{*,+, \S, \perp}$ and Ronald N. Zuckermann ${ }^{*}{ }^{\dagger}$
}

${ }^{\dagger}$ Molecular Foundry, ${ }^{\ddagger}$ Materials Sciences Division, and ${ }^{\S}$ Environmental Energy Technologies Division, Lawrence Berkeley National Laboratory, University of California, Berkeley, California 94720, United States

"Department of Materials Science and Engineering and ${ }^{\perp}$ Department of Chemical and Biomolecular Engineering, University of California, Berkeley, California 94720, United States

\section{Supporting Information}

ABSTRACT: Polymers that dissolve and conduct lithium ions are of great interest in the application of rechargeable lithium batteries. It is generally believed that the transport of ions in these systems is facilitated by rapid segmental motion typically found in rubbery, amorphous polymers. In this paper, we demonstrate that chemically identical ethyleneoxy-containing domains of a block copolymer exhibit comparable conductivities when in an amorphous or a crystalline state. An important feature of this study is the use of sequencedefined block copolypeptoids synthesized by submonomer solid-phase synthesis. Two structurally analogous ethyleneoxycontaining diblock copolypeptoids poly- $N$-(2-ethyl)hexylglycine-block-poly- $N$-2-(2-(2-methoxyethoxy)ethoxy)ethylglycine (pNeh-b-pNte) and poly- $N$-decylglycine-block-poly-N-2-(2-(2-methoxyethoxy)ethoxy)ethylglycine (pNdc- $b$-pNte) with 18 monomer units per block were synthesized. Both diblock copolypeptoids have the same conducting block, pNte, but different nonconducting blocks: $\mathrm{pNeh}$, which is amorphous, and $\mathrm{pNdc}$, which is crystalline. Both diblock copolypeptoids self-assemble into a lamellar morphology; however, $\mathrm{pNte}$ chains are amorphous in $\mathrm{pNeh}-b$-pNte and crystalline in $\mathrm{pNdc}-b$-pNte. This provides the platform for comparing lithium ion transport in amorphous and crystalline polymer domains that are otherwise similar.

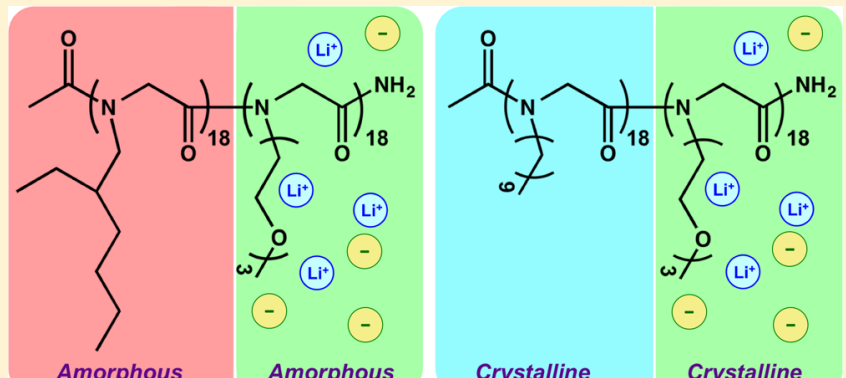

\begin{tabular}{l|l|l} 
Amorphous & Amorphous & Crystalline
\end{tabular}

\section{INTRODUCTION}

Block copolymers self-assemble into a number of nanostructured morphologies, including body-centered-cubic packed spheres, hexagonally packed cylinders, and lamellae. Traditional polymerization methods (e.g., controlled radical and anionic polymerization) offer limited levels of control over polydispersity and compositions of the copolymers. Polypeptoids are a family of comb-like sequence-defined polymers based on an $\mathrm{N}$ substituted glycine backbone. ${ }^{1,2}$ Unprecedented control over chain length and copolymer composition is enabled by the iterative solid-phase submonomer synthesis method., Although peptoids are structurally close to polypeptides, the absence of hydrogen-bond donors along the peptoid backbone results in a flexible chain with reduced interchain interactions and excellent thermal processability. ${ }^{5}$ In previous work, ${ }^{6,7}$ we studied two ethyleneoxy-containing block copolypeptoids pNeh- $b$-pNte (poly- $N$-(2-ethyl)hexylglycine-block-poly- $N$-2-(2(2-methoxyethoxy)ethoxy)ethylglycine) and pNdc- $b$-pNte (poly- $N$-decylglycine-block-poly-N-2-(2-(2-methoxyethoxy)ethoxy)ethylglycine). In this work, we focus on symmetric pNeh- $b$-pNte and pNdc- $b$-pNte constructs wherein the number of monomer units in each block was held fixed precisely at 18 . The chemical structures and phase behavior of these block copolymers are shown in Figure 1. The ordered morphology of both copolymers is lamellar with one important difference: at room temperature, both lamellar phases of pNeh- $b$-pNte are amorphous, while both of those of $\mathrm{pNdc}-b$-pNte are crystalline. In other words, the crystallinity of the $\mathrm{pNdc}$ chains induces crystallization of pNte. ${ }^{7}$ It is noteworthy that the pNte phase at room temperature can be either crystalline or amorphous, depending on the chemical structure of the block that it is attached to.

Ion-containing block copolymers are of interest for many applications, such as batteries, fuel cells, and photovoltaic devices. ${ }^{8-13}$ Here we focus on the effect of lithium salts on the thermodynamics and ionic conductivity of these two solid electrolyte systems. The conductivity of polymer electrolytes has been shown to depend crucially on the presence or absence of crystallinity. ${ }^{14-25}$ In the majority of publications it is reported that the conductivity of amorphous poly(ethylene oxide) (PEO) is much greater than that of crystalline PEO. ${ }^{14-19}$ Many have argued that the conductivity of semicrystalline salt/PEO mixtures is entirely due to the conductivity of the amorphous regions. On the other hand, Gadjourova et al. reported that the conductivity of oligomeric PEO (molecular weight $1 \mathrm{~kg} / \mathrm{mol}$ ) in the crystalline state with an $\mathrm{Li}$ to $\mathrm{O}$ molar ratio of $1: 6$ was about $10^{-7} \mathrm{~S} / \mathrm{cm}$ at room

Received: August 6, 2014

Published: September 24, 2014 


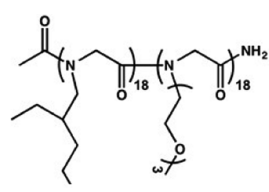

pNeh-b-pNte

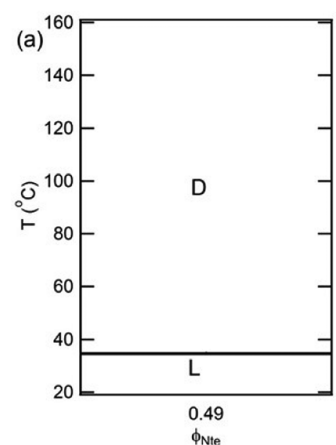

$\phi_{\text {Nte }}$

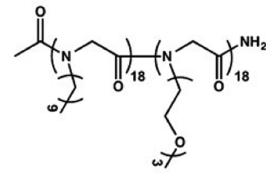

pNdc-b-pNte

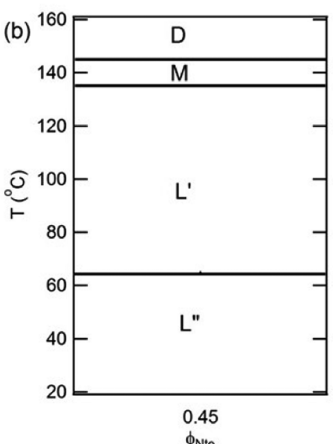

Q

Figure 1. Phase diagrams of two diblock copolypeptoids: pNeh- $b$ pNte (a) and pNdc- $b$-pNte (b), with identical chain length (18 monomers per block). D is the disordered phase, $\mathrm{L}$ is the lamellar phase, $M$ is metastable phase, $L^{\prime}$ is the lamellar phase with crystalline pNdc, and $\mathrm{L}^{\prime \prime}$ is the lamellar phase with two crystalline blocks, pNdc and pNte. The volume fractions of the pNte block in the copolymers, $\phi_{\mathrm{Nte}}$, are given.

temperature, while that of high molecular weight amorphous PEO (molecular weight $100 \mathrm{~kg} / \mathrm{mol}$ ) at the same salt concentration and temperature was $10^{-8} \mathrm{~S} / \mathrm{cm}^{20}$ Past comparisons of the conductivity of crystalline and amorphous polymers have invariably involved changing variables that have a strong effect on ion transport, such as polymer molecular weight, temperature, or thermal history. It is generally not trivial to isolate the effects of different parameters, e.g., see discussion by Henderson and Passerini. ${ }^{22}$ Here we address this important issue by studying the conductivity of chemically identical ethyleneoxy-containing polymers in both crystallinity and amorphous states. The pNeh- $b$-pNte and pNdc- $b$-pNte copolymer systems enable a direct comparison of the conductivity of crystalline and amorphous pNte domains at the same temperature, salt concentration, and chain length.

\section{RESULTS AND DISCUSSION}

The compositions of the polymer/salt mixtures studied in this paper are given in Table 1. In both polymers, the nonconducting polypeptoid blocks have similar chemical structures: the pNeh polypeptoid is amorphous due to the presence of a branched alkyl side chain, while the pNdc polypeptoid is crystalline due to the unbranched alkyl side chain. ${ }^{26}$ This subtle difference induces crystallization of pNte block in the pNdc- $b$-pNte block copolymer.

We first investigated the effect of added LiTFSI (LiN$\left(\mathrm{SO}_{2} \mathrm{CF}_{3}\right)_{2}$, lithium bis(trifluoromethanesulphonyl)imide) salt on the thermal properties of both block copolymers by DSC. The lack of melting peaks in the DSC data of pNeh- $b$-pNte/salt mixtures indicates that all the mixtures are amorphous (Figure $\mathrm{S} 1)$. This is expected because neat $\mathrm{pNeh}-b$-pNte is amorphous over the entire accessible temperature window and the addition of salt is known to reduce crystallinity. In Figure 2a, we show DSC data for pNdc- $b$-pNte as a function of salt concentration. At $r=0$, we see two peaks due to the melting of $\mathrm{pNte}\left(T_{\mathrm{m}, \mathrm{Nte}}=\right.$ $\left.60{ }^{\circ} \mathrm{C}\right)$ and $\mathrm{pNdc}\left(T_{\mathrm{m}, \mathrm{Ndc}}=140{ }^{\circ} \mathrm{C}\right)$ crystals. ${ }^{7}$ These melting temperatures are within the experimental range of melting

Table 1. Thermal and Structural Characteristics of Diblock Copolypeptoids pNeh- $b$-pNte and pNdc- $b$-pNte As Determined by SAXS ${ }^{a}$

\begin{tabular}{llccc} 
polypeptoids & \multicolumn{1}{c}{$r$} & $\phi_{\text {Nte }}$ & $d(\mathrm{~nm})$ & $T_{\text {ODT }}\left({ }^{\circ} \mathrm{C}\right)$ \\
& 0 & 0.49 & 7.1 & 35 \\
pNeh- $b$-Nte & 0.02 & 0.50 & 7.3 & 55 \\
& 0.04 & 0.51 & 7.6 & 65 \\
& 0.085 & 0.52 & 8.6 & 105 \\
& 0.16 & 0.55 & 9.2 & 110 \\
pNdc- $b$-pNte & & & & \\
& 0 & 0.45 & 10.8 & 140 \\
& 0.085 & 0.48 & 10.6 & 135 \\
& 0.16 & 0.51 & 9.2 & 145 \\
& 0.40 & 0.53 & 8.7 & 155 \\
& 0.60 & 0.65 & 10.7 & $>180$
\end{tabular}

${ }^{a} r$ is the molar ratio of cations to peptoid ethylene oxide moieties; $\phi_{\text {Nte }}$ is the volume fraction of pNte block; $d$ is the center-to-center distance between adjacent pNte lamellae, $d=2 \pi / q^{*}$; and $T_{\mathrm{ODT}}$ is the orderdisorder transition temperature.

temperatures of poly(ethylene oxide) and polyethylene homopolymers. This is not surprising as previous studies have shown that the crystallization of peptoids is dominated by their side chains. ${ }^{1,5}$ The addition of salt reduces the melting enthalpy $(\Delta H)$ of both blocks. At $r=0.085$, melting of both pNte and pNdc can be seen, although the pNte melting peak is reduced to a broad shoulder. At $r=0.16$, a small peak associated with the melting of $\mathrm{pNdc}$ domains and a very weak signature of the melting of pNte domains are evident. At $r=$ 0.20 , both melting peaks disappear. The melting temperature of the pNdc block is reduced from 140 to $120^{\circ} \mathrm{C}$ as $r$ is increased from 0 to 0.16 . It is evident that the addition of salt disrupts the crystallization of both $\mathrm{pNdc}$ and $\mathrm{pNte}$ domains.

WAXS analysis confirms the results of DSC, as shown in Figure $2 b$. In the neat block copolymer $(r=0),{ }^{25}$ a sharp primary peak at $q=q_{\mathrm{c}}{ }^{*}$ and higher-order peaks at $2 q_{\mathrm{c}}{ }^{*}, 3 q_{\mathrm{c}}{ }^{*}$, $5 q_{\mathrm{c}}^{*}$, and $6 q_{\mathrm{c}}^{*}$ reflect the spacing between the peptoid backbones, parallel to the side chains (c, shown in Figure 3 ). The peak at $q=q_{\mathrm{b}}$ reflects the distance between two adjacent side chains along the backbone ( $b$, shown in Figure 3$)$. These lattice parameters $b$ and $c$ apply to both pNdc and pNte crystals. A doublet at $q=q_{\mathrm{Ndc}}$ and $q_{\mathrm{Nte}}$ reflects the spacing between the peptoid backbones of $\mathrm{pNdc}$ and pNte block perpendicular to the side chains $\left(a_{\mathrm{Ndc}}\right.$ and $a_{\mathrm{Nte}}$, shown in Figure 3 ). All of the WAXS features persist as $r$ is increased from 0 to 0.16 and no change lattice observed in this range (Table 2). Our experiments thus far do not shed light on how the salt ions are incorporated into the pNte and pNdc crystals. As $r$ is further increased to 0.20 , all of the WAXS peaks disappear, indicating the absence of crystalline lamellae. Interestingly, the doublet at $q=q_{\mathrm{Ndc}}$ and $q_{\mathrm{Nte}}$ disappears simultaneously, indicating the intimate relationship between the two crystal phases.

While we have determined distances between some of the atoms in our crystalline peptoids (Figure 3), many aspects of the crystalline structure (e.g., space group) cannot be determined from the limited number of WAXS peaks, shown in Figure 2b. In related systems, crystallization of a peptoid hexamer with short alkyl side chains was studied by WAXS. ${ }^{5}$ Lee et al. also studied the crystalline structure of high molecular weight cyclic-poly $(N$-decylglycine $){ }^{26}$ Although additional 


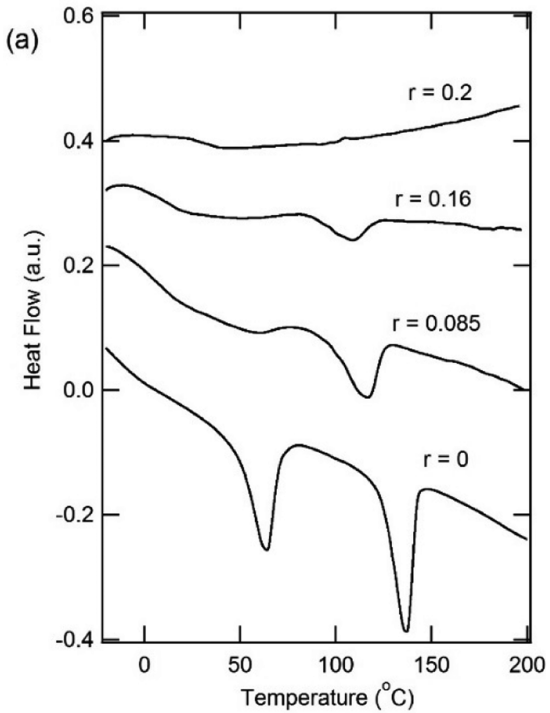

(b)

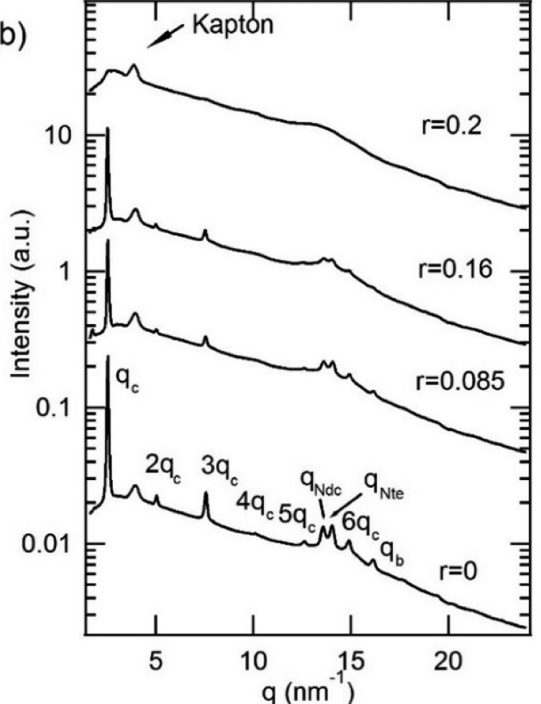

Figure 2. (a) DSC endotherms for pNdc- $b$-pNte at $r=0,0.085,0.16$, and 0.2. (b) WAXS patterns of pNdc- $b$-pNte at $r=0,0.085,0.16$, and 0.2 .

peaks were observed in both cases, there was still not enough evidence to assign a space group to the crystals. Further study is required to determine the exact crystal structure of $\mathrm{pNdc}-b$ pNte. Note that "chain-folded lamellae", found in conventional polymers, are absent; instead the peptoid chains are extended, and crystalline backbones are tilted relative to the normal to the block copolymer lamellae. $^{7}$

The data in Figure 2 indicate that LiTFSI is soluble in pNdc. This is not unexpected as some nitrogen-containing groups are known to also interact with lithium salts. ${ }^{27}$ To study these interactions, we incorporated LiTFSI salts into a pNdc homopolymer with 20 repeat units and repeated the DSC experiments. Data from these mixtures are summarized in Figure 4 where $\Delta H$, the enthalpy of melting of the pNdc peak normalized by the mass of $\mathrm{pNdc}$, is plotted as a function of salt concentration, $r_{\mathrm{N}}$. Here $r_{\mathrm{N}}$ is the molar ratio of lithium to the backbone CO-NR 2 groups. Also plotted in Figure 4 is $\Delta H$ corresponding to pNdc melting from $\mathrm{pNdc}-b$-pNte (the hightemperature melting peak in Figure 2a); in this case, $r_{\mathrm{N}}$ is the molar ratio of total lithium added to the $\mathrm{CO}-\mathrm{NR}_{2}$ groups in the pNdc block only. The $\Delta H$ values of $\mathrm{pNdc}$ homopolypeptoid

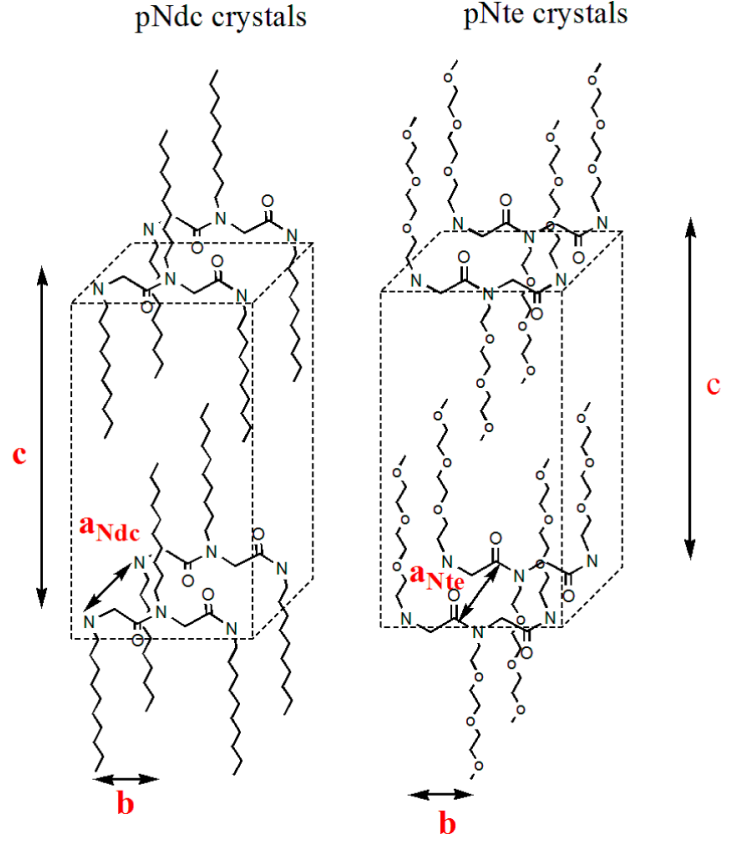

Figure 3. Schematic structure of two crystals, where $a_{\mathrm{Ndc}}$ is the interchain distance of the pNdc block, $a_{\mathrm{Nte}}$ is the interchain distance of pNte block, $b$ is the distance between adjacent monomer residues, and $c$ is the distance between peptoid chains. Note that $c$ remains $2.5 \mathrm{~nm}(c$ $\left.=2 \pi / q_{\mathrm{c}}{ }^{*}\right)$ from $r=0$ to 0.16 , twice the length of a fully extended decyl group (i.e., $12.4 \AA),{ }^{26}$ indicating interdigitation of side chains is unlikely. Dotted lines merely indicate the spacing between atoms.

$(21.7 \mathrm{~J} / \mathrm{g} \mathrm{pNdc})$ and $\mathrm{pNdc}$ block in $\mathrm{pNdc}-b$-pNte $(21.6 \mathrm{~J} / \mathrm{g}$ pNdc) are similar at $r_{\mathrm{N}}=0$. It is therefore reasonable to assume that the crystal structure of $\mathrm{pNdc}$ in the $\mathrm{pNdc}-b$-pNte copolymer is not effected by pNte. It is evident from Figure 4 that in both $\mathrm{pNdc}$ and $\mathrm{pNdc}-b$-pNte, the $\Delta H$ decreases linearly with $r_{\mathrm{N}}$, reaching a value of 0 at $r_{\mathrm{N}}=0.1$ and 0.5 , respectively. We assume that the salt concentration required to melt pNdc crystals is the same in the homopolymer and block copolymer. Thus, when $r_{\mathrm{N}}$ is 0.5 in the block copolymer, the local value of $r_{\mathrm{N}}$ in the pNdc-rich microphase must be 0.1 , and that in the pNte-rich microphase is 0.4. In other words, the partition coefficient of salt in the pNte micophase relative to the pNdc microphase is 4 . We were unable to quantify salt partitioning in the pNeh- $b$-pNte due to its amorphous structure. Lacking of a better alternative, and due to the similarity of the chemical structures, in the discussions below, we assume the same partition coefficient applies to both diblock copolymers. The major conclusions of this work are not qualitatively affected by this assumption.

The phase behavior of $\mathrm{pNdc}-b$-pNte as a function of salt concentration was investigated by SAXS. In Figure 5, we show SAXS data as a function of salt concentration, $r$, at room temperature. The neat polymer exhibits a broad primary scattering peak at $q^{*}=0.58 \mathrm{~nm}^{-1}$, which reflects the center-tocenter distance between adjacent pNte lamellae, $d=2 \pi / q^{*}(d=$ $10.8 \mathrm{~nm})$. The peak at $q=2.5 \mathrm{~nm}^{-1}$ is due to Bragg diffraction from pNte and pNdc crystals. The crystals disrupt the lamellar ordering resulting in the broad primary peak seen in Figure 5. The SAXS from the neat copolymer is discussed thoroughly in previous work. ${ }^{7}$ As $r$ is increased to 0.085 , a primary peak at $q^{*}$ $=0.59 \mathrm{~nm}^{-1}$ and a higher-order peak at $q=3 q^{*}$ are visible indicating the presence of the lamellar morphology. The expected peak at $2 q^{*}$ is missing because the estimated volume 
Table 2. Characteristics of the Diblock Copolypeptoid pNdc- $b$-pNte Obtained by WAXS and DSC

\begin{tabular}{|c|c|c|c|c|c|c|c|}
\hline$r$ & $\phi_{\mathrm{Nte}}$ & $c(\mathrm{~nm})$ & $a_{\mathrm{Ndc}}(\mathrm{nm})$ & $a_{\mathrm{Nte}}(\mathrm{nm})$ & $b(\mathrm{~nm})$ & $C_{\mathrm{r}, \mathrm{Ndc}}(\%)^{a}$ & $C_{\mathrm{r}, \mathrm{Nte}}(\%)^{c}$ \\
\hline 0 & 0.45 & 2.5 & 0.46 & 0.45 & 0.39 & $100 \%^{b}$ & $100 \%^{b}$ \\
\hline 0.085 & 0.48 & 2.5 & 0.46 & 0.45 & 0.38 & $60.1 \%$ & $74.6 \%$ \\
\hline 0.16 & 0.51 & 2.5 & 0.46 & 0.45 & 0.38 & $27.7 \%$ & $47.5 \%$ \\
\hline
\end{tabular}

${ }^{a}$ Percent crystallinity $C_{\mathrm{r}, \mathrm{Ndc}}$ is calculated by $\Delta H$ of pNdc block based on DSC data. ${ }^{b}$ The crystallinity at $r=0$ is assumed to be $100 \% .{ }^{c}$ Percent crystallinity $C_{\mathrm{r}, \mathrm{Nte}}$ is calculated based on the $C_{\mathrm{r}, \mathrm{Ndc}}$ and integrations of peaks $q=q_{\mathrm{Nte}}$ and $q_{\mathrm{Ndc}}$ from WAXS data. Directly integrating from DSC data was not possible as the pNte melting peaks are smeared over a wide temperature window in DSC.

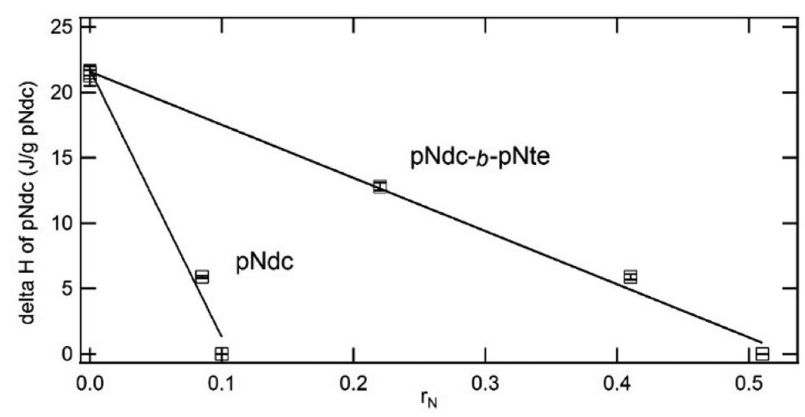

Figure 4. Plots of $\Delta H$ of the $\mathrm{pNdc}$ melting peak in $\mathrm{pNdc}$ homopolymer and pNdc- $b$-pNte diblock copolymer (normalized by mass of $\mathrm{pNdc}$ ) versus salt concentration, $r_{\mathrm{N}}$, the ratio of $\mathrm{Li}$ : CO-NR of $\mathrm{pNdc}$ homopolymer and $\mathrm{pNdc}$ in $\mathrm{pNdc}-b$-pNte and $\mathrm{pNdc}$ homopolymer.

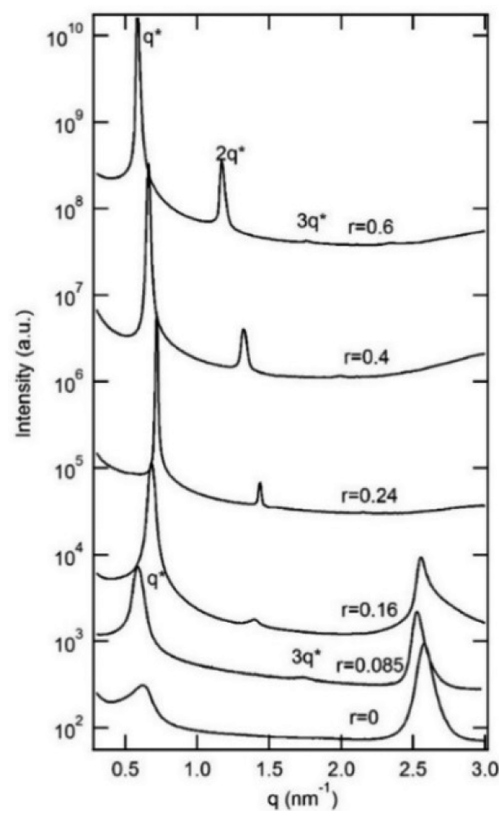

Figure 5. SAXS profiles at room temperature for $\mathrm{pNdc}-b$-pNte at $r=$ $0,0.085,0.16,0.24,0.4$, and 0.6 . Profiles are vertically offset for clarity.

fraction of pNte-rich microphase is in the vicinity of 0.5 . As $r$ is further increased to 0.16 , the higher order peak at $2 q^{*}$ is seen along with the crystal peak. At $r$ values of 0.24 and higher, the crystal peak disappears, and SAXS signatures of amorphous lamellae are seen. The lamellar structure was confirmed by TEM. In Figure 6 we show an unstained TEM micrograph obtained from the $r=0.085$ sample. The value of $d$ estimated by TEM is $10 \mathrm{~nm}$, consistent with $10.8 \mathrm{~nm}$ determined by SAXS.

SAXS profiles of the sample pNdc- $b$-pNte at $r=0.085$ as a function of temperature are shown in Figure $7 \mathrm{a}$. Increasing the

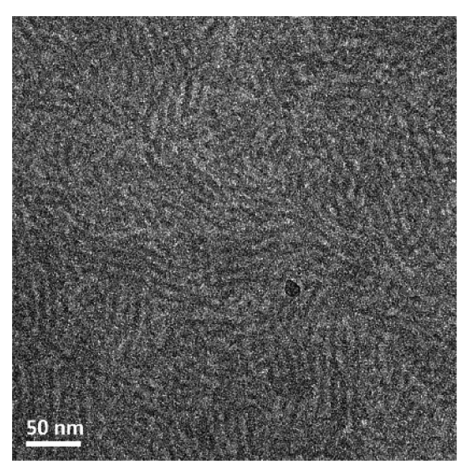

Figure 6. TEM image of $\mathrm{pNdc}-b$-pNte at $r=0.085$ showing the lamellar morphology at room temperature.
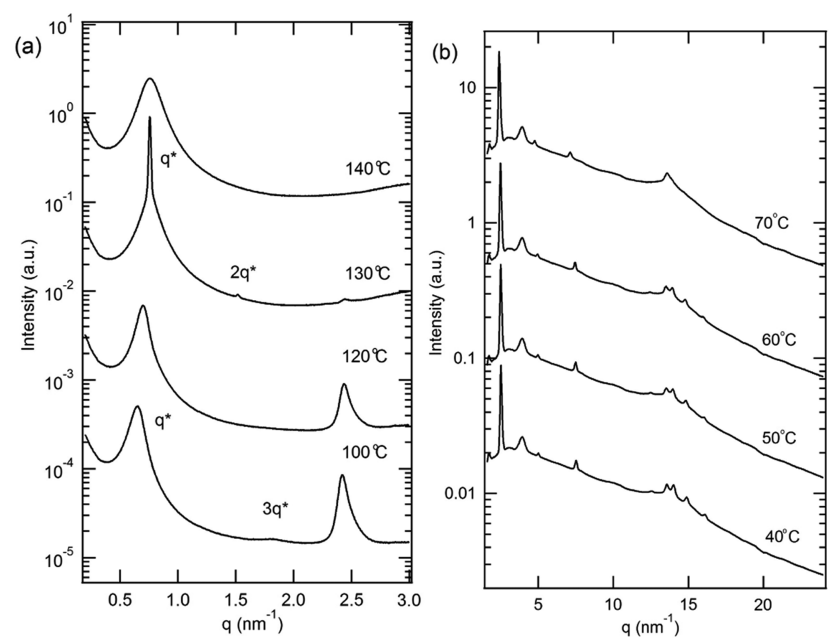

Figure 7. (a) SAXS intensity versus scattering vector, $q$, for $\mathrm{pNdc}-b$ pNte at $r=0.085$ at selected temperatures. (b)WAXS intensity versus scattering vector, $q$, for $\mathrm{pNdc}-b$-pNte at $r=0.085$ at selected temperatures. Profiles are vertically offset for clarity for both (a) and (b).

temperature to $120{ }^{\circ} \mathrm{C}$ at $r=0.085$ has virtually no effect on morphology. Further increase of the sample temperature to 130 ${ }^{\circ} \mathrm{C}$ results in a dramatic decrease in the intensity of the crystalline peak at $q=2.5 \mathrm{~nm}^{-1}$ and the appearance of a sharp primary peak at $q^{*}=0.76 \mathrm{~nm}^{-1}$ and a higher order peak at $2 q^{*}$. This indicates the coexistence of amorphous and crystalline lamellar phases, similar to those found in neat pNdc- $b$-pNte. ${ }^{7}$ Further increase of the sample temperature to $140{ }^{\circ} \mathrm{C}$ results in an order-disorder transition. The crystalline peak at $q=2.5$ $\mathrm{nm}^{-1}$ disappears, and the sharp primary peak at $q^{*}=0.76 \mathrm{~nm}^{-1}$ broadens abruptly at $140{ }^{\circ} \mathrm{C}$. The transitions seen in $\mathrm{pNdc}-b$ pNte at $r=0.085$ are very similar to those seen in neat $\mathrm{pNdc}-b$ pNte reported in ref 7 and are entirely reversible.

The SAXS data in Figure $7 \mathrm{a}$ do not indicate the state of the pNte-rich lamellae. We thus examined the temperature 
dependence of WAXS data from pNdc- $b$-pNte at $r=0.085$ (Figure $7 \mathrm{~b}$ ). A doublet at $q=q_{\mathrm{Ndc}}$ and $q_{\mathrm{Nte}}$ is evident at temperatures below $70{ }^{\circ} \mathrm{C}$. This indicates that the pNte-rich lamellae are crystalline at temperatures between 40 and $60{ }^{\circ} \mathrm{C}$.

Phase behavior as a function of salt and temperature were investigated by SAXS experiments on pNeh- $b$-pNte and pNdc$b$-pNte. The copolymers were examined at different salt concentrations, and the data obtained were similar to those in Figure 8 and our previous publications. ${ }^{6,7,28,29}$ These results
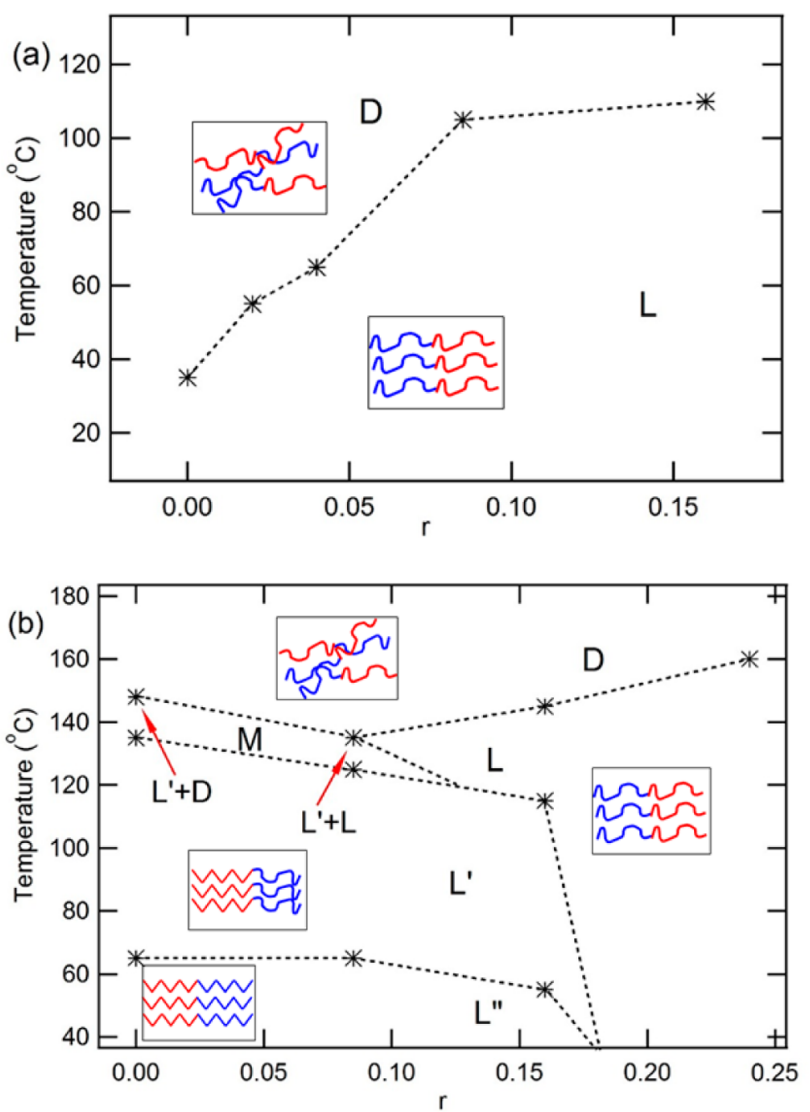

$\checkmark$ Crystalline $\mathrm{pNdc}$

$几$ Amorphous pNdc/pNeh

\rystalline pNte

$几$ Amorphous pNte

Figure 8. (a) Phase diagram of $\mathrm{pNeh}-b$-pNte at various temperatures and $r$, where $\mathrm{D}$ is the disordered phase and $\mathrm{L}$ is the lamellar phase. (b) Phase diagram of $\mathrm{pNdc}-b$-pNte at various temperatures and $r$, where D is the disordered phase, $\mathrm{M}$ is the metastable phase, $\mathrm{L}$ is the lamellar phase, $L^{\prime}$ is the lamellar phase with crystalline pNdc block, and $L^{\prime \prime}$ is the lamellar phase with two crystalline block. The red arrows indicate metastable phase of $\mathrm{L}^{\prime}$ and $\mathrm{D}$ at $r=0$ and $\mathrm{L}^{\prime}$ and $\mathrm{L}$ at $r=0.085$. Each marker represents a sample whose morphology was determined by SAXS. A phase boundary is bisected markers, indicative of the phase transition.

are summarized in Figure 8 . In the case of pNeh- $b$-pNte (Figure 8a), amorphous lamellar phases (L) are obtained at low temperature, and amorphous disordered phases (D) are obtained at high temperature. In the case of pNdc- $b$-pNte (Figure 8b), the phase behavior is rich, and it includes lamellar phases wherein both pNdc-rich and pNte-rich lamellae are crystalline $\left(\mathrm{L}^{\prime \prime}\right)$, lamellar phases wherein pNdc-rich lamellae are crystalline and pNte-rich lamellae are amorphous $\left(\mathrm{L}^{\prime}\right)$, and lamellar phases wherein both lamellae are amorphous (L) and disordered phases (D). The metastable phase (M) has coexisting $\mathrm{L}^{\prime}$ and $\mathrm{D}$ phases. The Gibbs phase rule requires metastable coexistence at all phase boundaries. In some cases, the temperature steps used in this work are too crude to quantify coexistence.

The characteristics of the pNte and pNdc crystals in the pNdc- $b$-pNte copolymers with and without salt are summarized in Table 2, where lattice parameters determined by WAXS and $\%$ crystallinity, determined by a combination of WAXS and DSC, at selected salt concentrations are provided. The addition of salt does not affect the lattice parameters, but it does lower the $\%$ crystallinity.

The order-to-disorder transition temperature $\left(T_{\mathrm{ODT}}\right)$ of $\mathrm{pNeh}-b$-pNte increases rapidly with salt concentration $r$ from 0.02 to 0.085 and levels off at large salt concentrations (Figure 8a). A similar effect has been seen in previous work on block copolymer polystyrene- $b$-poly(ethylene oxide)/salt mixtures. $^{28,30}$ This indicates that the effective repulsion between $\mathrm{pNeh}$-rich and pNte-rich lamellae is a strong function of salt concentration in the low salt concentration limit. It is customary to quantify this effect by an effective Flory-Huggins interaction parameter, $\chi_{\text {eff }}{ }^{30}$ It is believed that the phase behavior of $\mathrm{pNeh}-b$-pNte/salt mixtures reflects the dependence of $\chi_{\text {eff }}$ on $r$. The dependence of $T_{\mathrm{ODT}}$ on $r$ of pNdc- $b$-pNte is very different from that of $\mathrm{pNeh}-b$-pNte in spite of the similarity of chemical structure and the fact that the chain length is identical. The addition of salt leads to a decrease of $T_{\text {ODT }}$ followed by a modest linear increase. Microphase separation in pNdc- $b$-pNte is dominated by the crystalline nature of the constituent blocks. The addition of salt has a modest effect on both the $L^{\prime}$ to $L^{\prime \prime}$ transition temperature and the melting of pNte crystals in the $\mathrm{L}^{\prime}$ phase in the range $0.00 \leq$ $r \leq 0.16$. An abrupt transition is seen between $r=0.16$ and 0.20 . At $r=0.20$ and higher, both pNte-rich and pNdc-rich lamellae are amorphous. We posit that this is a signature of induced crystallization. ${ }^{7}$ The formation of pNte crystals in block copolymer pNdc- $b$-pNte is induced by crystallization of $\mathrm{pNdc}$ chains. At $r=0.20$, the $\mathrm{pNdc}$ is disrupted, and this in turn disrupts pNte crystallization.

There are interesting differences in the dependence of domain spacing on salt concentration and temperature of pNdc- $b$-pNte and pNeh- $b$-pNte. Figure 9a shows the dependence of $d$ on $r$ at room temperature. We see that $d$ increases from 7.1 to $9.2 \mathrm{~nm}$ in $\mathrm{pNeh}-b$-pNte, as $r$ increases from 0 to 0.16 . This is generally consistent with the notion that $\chi_{\text {eff }}$ increases with $r$. The domain spacing $d$ of pNdc- $b$-pNte shows a different dependence on salt concentration. It declines from 10.8 to $8.7 \mathrm{~nm}$ as $r$ increases from 0 to 0.24 . However, as salt concentration increases further from 0.24 to $0.6, d$ increases monotonically (we do not report the phase behavior of $\mathrm{pNdc}-b$ pNte at $r>0.24$ in Figure $8 \mathrm{~b}$ because the amorphous lamellae are obtained at all salt concentrations and temperatures). As established in Table 2 , the crystalline lattice parameters $\left(a_{\mathrm{Nd} o}\right.$ $\left.a_{\mathrm{Nte}}, b, c\right)$ are largely unaffected by salt. In spite of this, there is a significant decrease in $d$ of the $\mathrm{L}^{\prime \prime}$ phase with increasing $r$. At $r$ values where $\mathrm{pNdc}-b$-pNte is amorphous $(r>0.24), d$ increases with increasing $r$, and the rate of this increase is similar to that seen in amorphous pNeh- $b$-pNte. In Figure $9 b$, we show the temperature dependence of $d$ of both systems at $r=0.085$. In the amorphous sample, $d$ remains constant with increasing temperature, while in crystalline sample, $d$ deceases with increasing temperature. Similar effects are observed in amorphous and crystalline block copolymers in absence of salt. $^{31-35}$ 

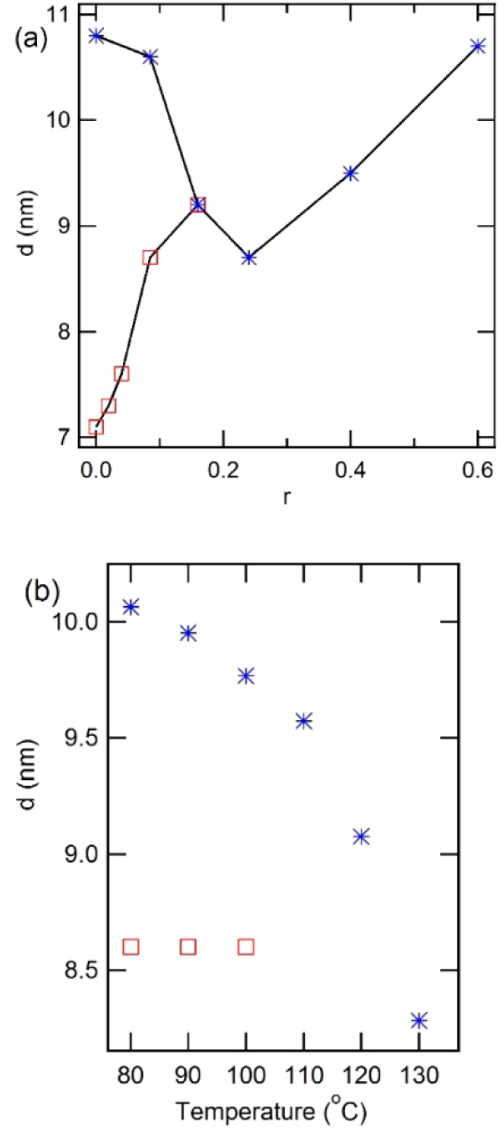

Figure 9. Effect of salt and temperature on the lamellar spacing; $d$ value plots as a function (a) of $r$ ratios and (b) of temperatures at $r=$ 0.085 . Squares represents $\mathrm{pNeh}-b$-pNte and asterisks represents $\mathrm{pNdc}-$ $b$-pNte.

The ionic conductivity $(\sigma)$ for both block copolymers is shown in Figure 10 in the usual $\sigma$ versus 1000/T format. ${ }^{28}$ We measured the ionic conductivities from 40 to $90{ }^{\circ} \mathrm{C}$ at $r=0.085$ and 0.16 . In this range, both diblock copolymers are lamellar but the pNte-rich domains are semi-crystalline or amorphous in $\mathrm{pNdc}-b$-pNte, depending on the temperature. In contrast, the

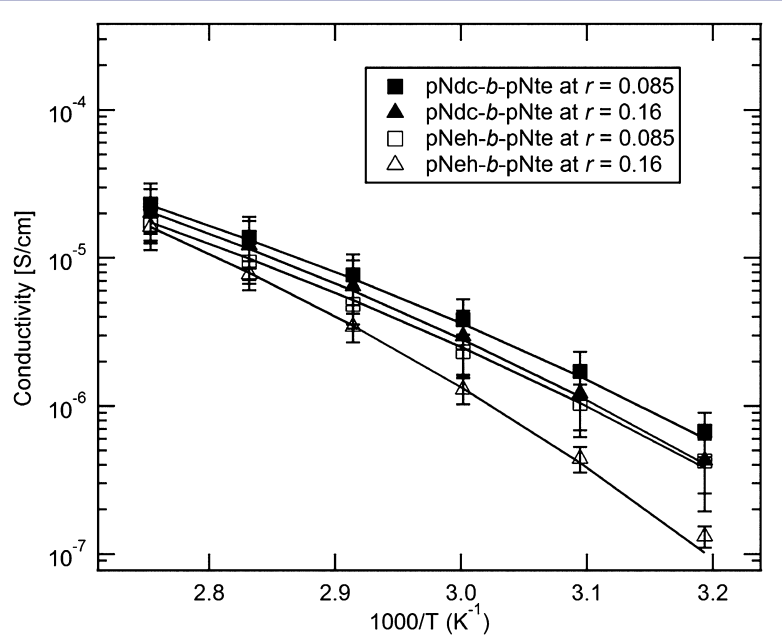

Figure 10. Ionic conductivity plots of pNeh- $b$-pNte and pNdc- $b$-pNte as a function of temperature at salt concentrations of $r=0.085$ and 0.16 . The lines through the data points are VTF fits. lamellae are amorphous in pNeh-b-pNte regardless of temperature. This enables a direct comparison of the conductivity of crystalline and amorphous $\mathrm{pNte}$ domains. Note that the relative crystallinities $\left(C_{\mathrm{r}, \mathrm{Nte}}\right)$ of pNte in block copolymer at $r=0.085$ and 0.16 are 74.6 and $47.5 \%$ (Table 2). One expects the amorphous lamellae to have higher conductivity based on the conclusions in a majority of publications..$^{15-20}$ The data in Figure 10 do not support this expectation; similar conductivities are obtained in $\mathrm{pNeh}-b$-pNte and $\mathrm{pNdc}-b$-pNte at the same salt concentration and temperature. In addition, the melting of the pNte crystals in pNdc- $b$-pNte at $\mathrm{L}^{\prime \prime}$ to $\mathrm{L}^{\prime}$ does not cause a shift in the $\sigma$ versus $1000 / T$ plot. The curves in Figure 10 are fits of the data to Vogel-Tamman-Fulcher (VTF):

$$
\sigma=A \exp \left\{(-B) /\left(R\left(T-T_{0}\right)\right\}\right.
$$

where $A$ is a constant proportional to the number of charge carriers, $B$ is equivalent to the activation energy for ion motion, $R$ is the gas constant, $T$ is the temperature, and $T_{0}$ is a reference temperature which is $T_{\mathrm{g}}-83 \mathrm{~K}$. Table $\mathrm{S} 1$ in Supporting Information gives the VTF fit parameters used to obtain curves in Figure 10. In their measurement of conductivity through crystalline PEO oligomers, MacGlashan et al. obtained an exponential relationship between $\sigma$ and $1 / T^{21}$ In contrast, we obtained slightly curved plots of $\sigma$ versus $1 / T$ for the case of $\mathrm{pNdc}-b$-pNte (Figure 10). This suggests that the mechanisms of ion transport in crystalline pNte-rich lamellae and oligomeric PEO may be different.

An interesting feature in our system is the partitioning of the salt in both lamellae. We have established that the salt partition coefficient is $1: 4$ for $\mathrm{pNdc}-b$-pNte. Thus, the expected salt concentration in pNte phase, $r_{\text {real }}=0.8 r$. In other words, the real salt concentrations in the pNte-rich lamellae in $\mathrm{pNdc}-b$ pNte samples described in Figure 10 are 0.068 and 0.128, respectively. Lacking a better alternative, we assume that the same partition coefficient holds for $\mathrm{pNeh}-b$-pNte.

Interpretation of the data in Figure 10 requires quantification of the conductivity of salt-containing pNte, $\mathrm{pNeh}$, and $\mathrm{pNdc}$ homopolymers. This is done in Figure 10 where $\sigma$ is plotted as a function of $1000 / T$ for these systems. The salt concentration in $\mathrm{pNdc}_{20}$ and $\mathrm{pNeh}_{20}$ was fixed at $r_{\mathrm{N}}=0.085$, while the salt concentration in pNte systems was fixed at $r=0.068$. The $\mathrm{pNte}_{20}$ data present in Figure 11 were obtained by interpolation of measurements of conductivity versus salt concentration reported in previous work ${ }^{36}$ and Figure S5 in Supporting Information (the other data in Figure 11 were measured directly). It is clear that the conductivity of $\mathrm{pNte} / \mathrm{salt}$ mixtures is a weak function of chain length. Furthermore, at a given temperature (e.g., $1000 / T=2.8$ ), the conductivity of pNte is about $10^{4}$ times higher than that of $\mathrm{pNdc}$ and $\mathrm{pNeh}$. To a good approximation, the pNeh-rich and pNdc-rich lamellae are insulating in spite of the presence of the salt.

To focus on this effect of crystallization on conductivity, we define the normalized conductivity, $\sigma_{n}$, using the equation:

$$
\sigma_{n}=\sigma /\left(\phi_{\mathrm{c}} \sigma_{\mathrm{am}, \mathrm{nte}}\right)
$$

where $\sigma_{\text {am,nte }}$ is the measured conductivity of the $\mathrm{pNte}_{20} / \mathrm{salt}$ mixture with appropriate salt concentration, $r=0.068$ and $r=$ 0.128 for $\sigma$ measured at $r=0.085$ and $r=0.16$, respectively (this accounts for salt partitioning, and $\phi_{\mathrm{c}}$ is volume fraction of the conducting pNte-rich block). The temperature-dependent values of $\sigma_{\text {am,nte }}(r=0.068)$ and $\sigma_{\text {am,nte }}(r=0.128)$ are shown in Figure 11 and Figure S6 in the Supporting Information. Figure 


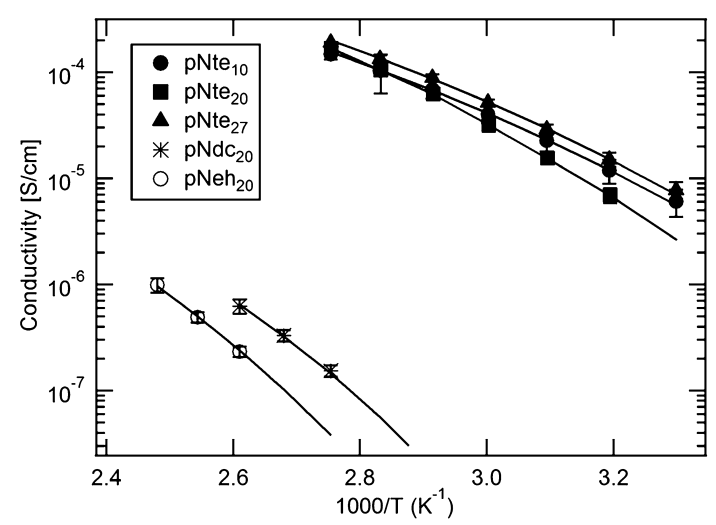

Figure 11. Ionic conductivity plots of homopolypeptoids, including: $\mathrm{pNte}_{10}, \mathrm{pNte}_{20}$, and $\mathrm{pNte}_{27}$ as a function of temperature from 30 to $100{ }^{\circ} \mathrm{C}$ at a salt concentration of $r=0.068$ and $\mathrm{pNdc}_{20}$ and $\mathrm{pNeh}_{20}$ as a function of temperature from 90 to $110^{\circ} \mathrm{C}$ and from 110 to $130{ }^{\circ} \mathrm{C}$, respectively, at a salt concentration of $r_{\mathrm{N}}=0.085$. The lines through the data points are VTF fits. Ionic conductivity plot of $\mathrm{pNte}_{20}$ is obtained by linear interpolation of measurement in Figure S5 in Supporting Information.

12 shows temperature dependence of $\sigma_{\mathrm{n}}$. The percent crystallinity of pNte-rich crystals at each temperature is also

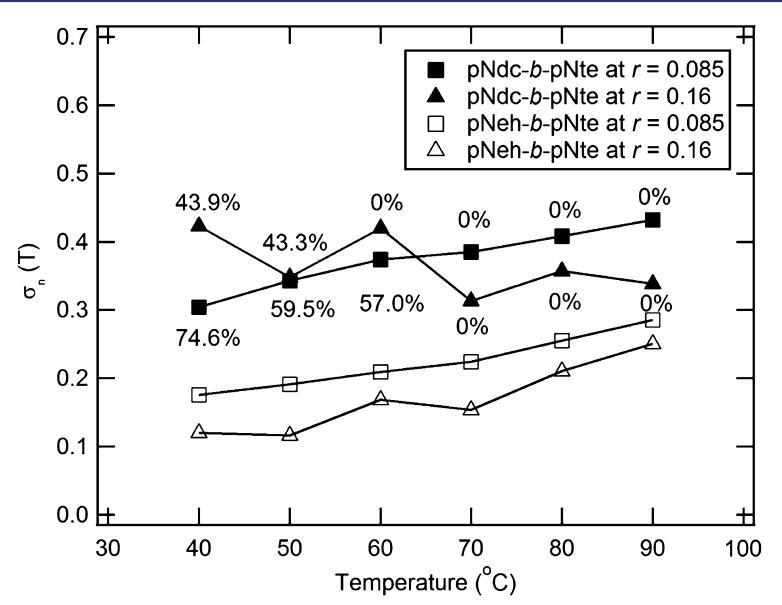

Figure 12. Plots of $\sigma_{n}$ of pNeh- $b$-pNte and pNdc- $b$-pNte as a function of temperature at $r=0.085$ and 0.16 . The $r$ value is corrected with $r_{\text {Nte-real }}$ that partitions into pNte blocks. The number at selected temperatures indicate that the percent crystallinity of the pNte domains in pNdc- $b$-pNte as determined by WAXS and DSC.

indicated in Figure 12. These percentages are based on the measured crystallinity of neat pNdc- $b$-pNte (see Table 2 ). Note that the pNte-rich lamellae in $\mathrm{pNeh}-b$-pNte are amorphous at all temperatures. It is evident in Figure 12 that there is no discernible effect of the melting of pNte crystals on $\sigma_{n}$ of pNdc$b$-pNte; values between 0.25 and 0.45 are obtained. Normalized conductivity of the fully amorphous pNeh- $b$-pNte systems ranges from 0.1 to 0.3 . The quantitative difference between the different systems seen in Figure 12 may be related to effects such as connectivity of conducting lamellae, the extent to which nonconducting segments are excluded from the conducting domains, interfacial width, etc. The main conclusion from Figure 12 is that crystalline and amorphous pNte domains have comparable intrinsic conductivity at the same chain length, temperature, and salt concentration. Overwhelming evidence indicates that crystals of high molecular weight linear PEO are nonconductive. ${ }^{11-13,37-41}$ This conclusion is based on data obtained from semicrystalline samples with coexisting crystalline and amorphous regions. Because polymer crystallization occurs slowly, the measured conductivity drifts with time, and it is impossible to clearly quantify the intrinsic conductivity of crystalline and amorphous. ${ }^{22}$ Our use of pNdc- $b$-pNte and pNeh- $b$-pNte circumvents this problem as crystalline and amorphous pNte chains are obtained at the same temperature due to templating or lack thereof by the nonconducting block.

\section{CONCLUSION}

The structure-conductivity relationships of two types of monodisperse diblock copolypeptoids doped with LiTFSI have been systematically studied. The two diblock copolymers are $\mathrm{pNeh}-b$-pNte and $\mathrm{pNdc}-b$-pNte that contain the same pNte block that dissolves the salt and conducts ions and similar nonconducting blocks: $\mathrm{pNeh}$ and $\mathrm{pNdc}$. The chain length is fixed at 18 for each block. However, their thermal properties are very different: at room temperature, $\mathrm{pNeh}-b$-pNte selfassembles into a lamellar structure with both amorphous microphases, while pNdc- $b$-pNte self-assembles into a lamellar structure with both crystalline phases. This allows for a direct comparison of the conductivity of crystalline and amorphous pNte domains. It is observed the lithium salt can partition into the domain of pNdc blocks and disrupt its crystallization, as indicated by DSC and WAXS. The partition coefficient of LiTFSI is 4:1 for pNte:pNdc. In general, the addition of salt can increase the strength of segregation as indicated by increasing $d$ and $T_{\mathrm{ODT}}$. Although the two copolymer systems have different thermal properties, both systems only exhibit lamellar and disordered structures. Mixtures of pNdc- $b$-pNte and salt exhibit a variety of lamellar phases including two crystalline microphases $\left(\mathrm{L}^{\prime \prime}\right)$, one crystalline and one amorphous microphase $\left(\mathrm{L}^{\prime}\right)$, and two amorphous microphases (L). Temperaturedependent ionic conductivity of pNeh- $b$-pNte and pNdc- $b$ pNte at $r$ of 0.085 and 0.16 were consistent with the VTF equation over the entire experimental window. The intrinsic conductivity of crystalline pNte lamellae in $\mathrm{pNdc}-b$-pNte is comparable to that of amorphous pNte in pNeh- $b$-pNte, at the same temperature and at two widely different salt concentrations. The reason for this observation is undoubtedly related to the manner in which the lithium salt is incorporated into the pNte crystalline phase. While considerable work remains to be done, our work represents one of a series of steps ${ }^{20-25}$ toward establishing the efficacy of crystalline polymers for lithium ion transport in batteries.

\section{ASSOCIATED CONTENT}

\section{S Supporting Information}

The experimental part and DSC of block copolypeptoid pNte$b$-pNeh and homopolypeptoid pNdc, plots of volume of homopolypeptoids/LiTFSI versus $r$, conductivity data of pNte $_{20}$ and $T_{\mathrm{g}}$ of block copolypeptoids at different $r$. This material is available free of charge via the Internet at http:// pubs.acs.org.

\section{AUTHOR INFORMATION}

\section{Corresponding Authors}

nbalsara@berkeley.edu rnzuckermann@lbl.gov

\section{Notes}

The authors declare no competing financial interest. 


\section{ACKNOWLEDGMENTS}

Funding for this work was provided by the Soft Matter Electron Microscopy Program, supported by the Office of Science, Office of Basic Energy Science, U.S. Department of Energy, under contract no. DE-AC02-05CH11231. The work was carried out at the Molecular Foundry at Lawrence Berkeley National Laboratory, supported by the Office of Science, Office of Basic Energy Science, U.S. Department of Energy, under contract no. DE-AC02-05CH11231. We thank Dr. Lei Zhang for his experimental help on the project.

\section{REFERENCES}

(1) Sun, J.; Zuckermann, R. N. ACS Nano 2013, 7, 4715-4732.

(2) Simon, R. J.; Kania, R. S.; Zuckermann, R. N.; Huebner, V. D.; Jewell, D. A.; Banville, S.; Ng, S.; Wang, L.; Rosenberg, S.; Marlowe, C. K. Proc. Natl. Acad. Sci. U.S.A. 1992, 89, 9367-9371.

(3) Zuckermann, R. N.; Kerr, J. M.; Kent, S. B. H.; Moos, W. H. J. Am. Chem. Soc. 1992, 114, 10646-10647.

(4) Tran, H.; Gael, S. L.; Connolly, M. D.; Zuckermann, R. N. J. Visualized Exp. 2011, 3373.

(5) Rosales, A. M.; Murnen, H. K.; Zuckermann, R. N.; Segalman, R. A. Macromolecules 2010, 43, 5627-5636.

(6) Sun, J.; Teran, A. A.; Liao, X.; Balsara, N. P.; Zuckermann, R. N. J. Am. Chem. Soc. 2013, 135, 14119-14124.

(7) Sun, J.; Teran, A. A.; Liao, X.; Balsara, N. P.; Zuckermann, R. N. J. Am. Chem. Soc. 2014, 136, 2070-2077.

(8) Park, M. J.; Nedoma, A. J.; Geissler, P. L.; Balsara, N. P.; Jackson,

A.; Cookson, D. Macromolecules 2008, 41, 2271-2277.

(9) Tsori, Y.; Tournilhac, F.; Leibler, L. Macromolecules 2003, 36, 5873-5877.

(10) Gomez, E. D.; Panday, A.; Feng, E. H.; Chen, V.; Stone, G. M.; Minor, A. M.; Kisielowski, C.; Downing, K. H.; Borodin, O.; Smith, G. D. Nano Lett. 2009, 9, 1212-1216.

(11) Young, W.-S.; Epps Iii, T. H. Macromolecules 2009, 42, 26722678.

(12) Naidu, S.; Ahn, H.; Gong, J.; Kim, B.; Ryu, D. Y. Macromolecules 2011, 44, 6085-6093.

(13) Nakamura, I.; Balsara, N. P.; Wang, Z.-G. Phys. Rev. Lett. 2011, 107, 198301.

(14) Berthier, C.; Gorecki, W.; Minier, M.; Armand, M. B.; Chabagno, J. M.; Rigaud, P. Solid State Ionics 1983, 11, 91-95.

(15) Ratner, M. A.; Shriver, D. F. Chem. Rev. 1988, 88, 109-124.

(16) Gray, F. M. RSC Materials Monographs; The Royal Society of Chemsitry: Cambridge, 1997.

(17) Abraham, K. M. Applications of Electroactive Polymers; Scrosati, B., Ed.; Chapman and Hall: London, 1993; p 182.

(18) Armand, M. Adv. Mater. 1990, 2, 278-286.

(19) Polymer electrolyte reviews; MacCallum, J. R.; Vincent, C. A., Eds.; Elsevier Science: New York, 1989; Vol. 2.

(20) Gadjourova, Z.; Andreev, Y. G.; Tunstall, D. P.; Bruce, P. G. Nature 2001, 412, 520-523.

(21) MacGlashan, G. S.; Andreev, Y. G.; Bruce, P. G. Nature 1999, $398,792-794$.

(22) Henderson, W. A.; Passerini, S. Electrochem. Commun. 2003, 5, $575-578$.

(23) Cheng, S.; Smith, D. M.; Li, C. Y. Macromolecules 2014, 47, 3978-3986.

(24) Maranas, J. K. In Dynamics of Soft Matter; Springer: Dordrecht, 2012; pp 123-143.

(25) Fullerton-Shirey, S. K.; Maranas, J. K. Macromolecules 2009, 42, $2142-2156$.

(26) Lee, C. U.; Smart, T. P.; Guo, L.; Epps, T. H.; Zhang, D. Macromolecules 2011, 44, 9574-9585.

(27) Alarco, P. J.; Abu-Lebdeh, Y.; Abouimrane, A.; Armand, M. Nat. Mater. 2004, 3, 476-481.
(28) Singh, M.; Odusanya, O.; Wilmes, G. M.; Eitouni, H. B.; Gomez, E. D.; Patel, A. J.; Chen, V. L.; Park, M. J.; Fragouli, P.; Iatrou, H. Macromolecules 2007, 40, 4578-4585.

(29) Panday, A.; Mullin, S.; Gomez, E. D.; Wanakule, N.; Chen, V. L.; Hexemer, A.; Pople, J.; Balsara, N. P. Macromolecules 2009, 42, $4632-4637$.

(30) Teran, A. A.; Balsara, N. P. J. Phys. Chem. B 2013, 118, 4-17.

(31) Wanakule, N. S.; Panday, A.; Mullin, S. A.; Gann, E.; Hexemer, A.; Balsara, N. P. Macromolecules 2009, 42, 5642-5651.

(32) Quiram, D. J.; Richard, A.; Marchand, G. R. Macromolecules 1997, 30, 4551-4558.

(33) Hamley, I. W.; Fairclough, J. P. A.; Bates, F. S.; Ryan, A. J. Polymer 1998, 39, 1429-1437.

(34) Mai, S. M.; Fairclough, J. P. A.; Viras, K.; Gorry, P. A.; Hamley, I. W.; Ryan, A. J.; Booth, C. Macromolecules 1997, 30, 8392-8400.

(35) Zhu, L.; Cheng, S. Z. D.; Calhoun, B. H.; Ge, Q.; Quirk, R. P.; Thomas, E. L.; Hsiao, B. S.; Yeh, F.; Lotz, B. Polymer 2001, 42, 58295839.

(36) Sun, J.; Stone, G. M.; Balsara, N. P.; Zuckermann, R. N. Macromolecules 2012, 45, 5151-5156.

(37) Fu, Y.; Pathmanathan, K.; Stevens, J. R. J. Chem. Phys. 1991, 94, $6323-6329$.

(38) Maccallum, J. R.; Smith, M. J.; Vincent, C. A. Solid State Ionics 1984, 11, 307-312.

(39) Cowie, J. M. G.; Sadaghianizadeh, K. Solid State Ionics 1990, 42, 243-249.

(40) Labreche, C.; Levesque, I.; Prud'Homme, J. Macromolecules 1996, 29, 7795-7801.

(41) Scrosati, B.; Croce, F.; Persi, L. J. Electrochem. Soc. 2000, 147, $1718-1721$. 